\title{
Modelling of cellular structures obtained by X-ray phase contrast imaging
}

\author{
E.A. Baglaeva, S.G. Tsapko, I.V.Tsapko \\ Institute of Cybernetics \\ National Research Tomsk Polytechnic University \\ Tomsk, Russia \\ eab14@tpu.ru
}

\author{
A.A. Ershov \\ Institute of Photonic research and Synchrotron radiation \\ Karlsruhe Institute of Technology \\ Karlsruhe, Germany
}

\begin{abstract}
Nowadays computed tomography provides the possibility to image internal cellular structures of embryos. A major challenge is a high accuracy image segmentation of tissues and individual cells. The process of manual image segmentation is time consuming and error prone. It can be partially replaced or augmented by cell modelling techniques developed by computer scientists based on biological, physiological and statistical properties of real embryos.
\end{abstract}

Keywords-imaging methods; microscopy; X-ray phase contrast; image segmentation; modelling of cellular structures

\section{INTRODUCTION}

At the recent times, embryogenesis became one of the promising areas in cell biology, developmental biology, genetics and toxicology. Despite the fact that the processes of embryogenesis have been studied since the last century, latest methods of computed tomography allow to obtain new knowledge about the processes of embryo development and allow to create detailed three-dimensional models of its internal structure.

Xenopus Laevis embryos are often used as model organisms for tracking ontogenesis stages. Ease of maintenance and manipulations made this organism an important object of embryology and developmental biology. Using Xenopus Laevis embryos, scientists can clearly trace the processes of displacement and differentiation of cells, forming tissues and individual organs [1].

Images, used for a detailed study of the embryo cellular structure, were obtained at European Synchrotron Research Facility (ESRF) and the Advanced Photon Source (APS) using $\mathrm{X}$-Ray phase contrast [2] techniques.

After tomographic reconstruction was completed, threedimensional image has to be processed to remove noise and artifacts to reach a result that allows distinguishing the cells and cellular structures (Fig. 1).

However, manual processing of the acquired images to obtain detailed three-dimensional model is a time-consuming task and, therefore, may be replaced or supplemented by the modelling techniques.
At the initial stage of cell modelling, cell data are collected: shape, size and location, then the segmentation of individual cell types is performed. The next step is the generating cellular structures based on the analysed statistics and geometric models of the segmented cells. Final stage is a visualization of a three-dimensional model. The paper pays special attention to methods of imaging and cell segmentation.

\section{REVIEW ON IMAGING TECHNIQUES}

Obtaining images of the cell structures can be performed using the opportunities of microscopy and computer tomography. Application of these imaging techniques depends on the size of the studied structures, their physical and optical properties.

For getting the information about the cell structure and intracellular processes, the following methods can be clarified: elemental, chemical, molecular and morphological analysis [3].

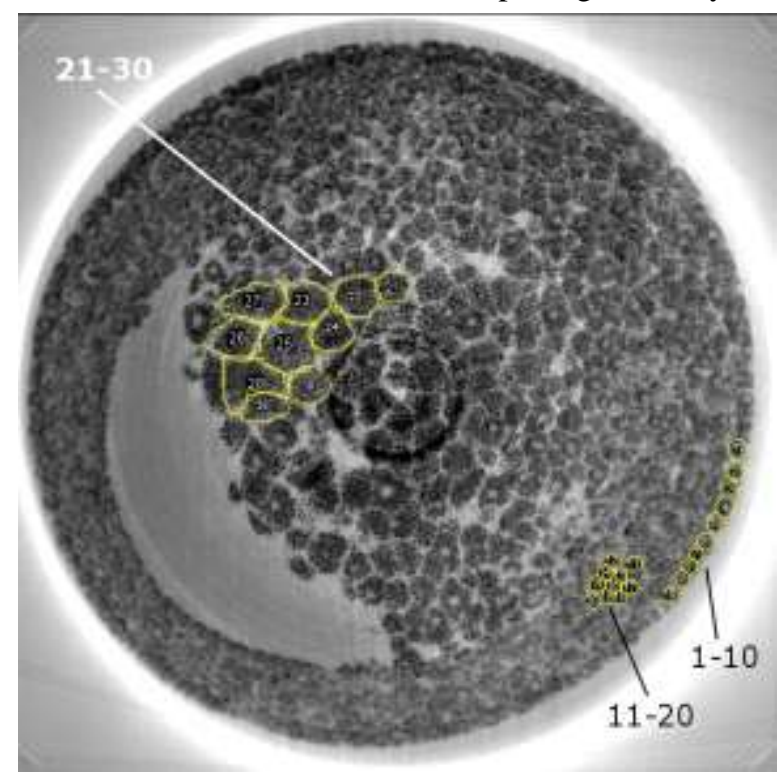

Fig. 1. Cut of the embryo obtained using X-Ray phase contrast (partially isolated cells are in the areas of: 1-10 - ectoderm, 11-20 - mesoderm, 21-30 endoderm 
A fluorescent microscopy implies that molecules of a particular type must be labelled with a fluorophore, and unlabelled molecules can not be fixed on the image. Confocal microscopy can significantly improve the quality of the images, as it provides a significant contrast in comparison with a conventional microscope.

For collecting cell morphological information (structure and shape), following methods are used: optical microscopy [6], X-ray phase contrast [7], atomic force microscopy [8], ellipsometry imaging [9] and the digital holography imaging [10].

Optical microscopy is one of the most common methods of obtaining information about the cell shape, but one of its major limitation is the length of the light wave. In fact, optical microscopes are used for quality control of cell structures (shape, viability, growth). The maximum resolution of the optical microscope is achieved using shortwave UV light of $200 \mathrm{~nm}[4,6]$.

Resolution of X-ray microscopy is between the resolution of optical and electron microscopy which allows to see detailed intracellular structure. The advantage of the X-ray to electron microscopy is that the use of $\mathrm{X}$-ray radiation allows observation of cellular structures at their natural state, i.e. in the cell culture. Moreover, application of phase contrast in combination with the use of X-ray tomography (X-ray phase contrast) provides the possibility to construct high-detailed three-dimensional models of cells and intracellular structures [3].

Atomic force microscopy provides resolution less than 1 $\mathrm{nm}$, which is more than 1000 times higher than the optical microscope does [8].

Ellipsometric methods are based on changes in the polarization of light after its interaction with the surface boundaries of different media (solid, liquid, gaseous) [9].

Digital holographic microscopy is a recently developed technique, which is suitable for the cell research, because it enables real-time reproducing of high-detailed threedimensional images. It provides a resolution of $290 \mathrm{~nm}[4,10]$.

Elemental analysis determines which chemical elements comprises the analyte. Cell elemental analysis is carried out using the following techniques: X-Ray fluorescence microscopy, PIXE (particle-induced X-ray emission microscopy) and SIMS (secondary ion mass spectrometry microscopy) [3].

Visualization of cell chemical processes is carried using the following methods: FTIR (Fourier-transform infrared), Raman microscopy, coherent anti-stokes Raman scaterring microscopy, scattering near-field optical microscopy [3].

\section{Methods of Cell Segmentation}

Segmentation is a process of isolation, visualization and quantification of the individual cell components, such as the cytoskeleton or cell organelles while the tomographic reconstruction is implemented.
Segmentation can be performed manually or semiautomatically.

Manual segmentation is performed interactively by identifying areas of interest on the basis of the threshold values of absorption or by visual inspection.

In addition, the cell may be segmented using automated methods. Software tools, solving this problem, use the absorption coefficients and the gradient between the regions in the reconstruction. There is a variety of standard approaches of image processing, such as edge detection and smoothing.

Allocation and delineation of required areas is performed starting with a user-defined threshold values of absorption or other user-defined characteristics, and using growth regions algorithms [11].

Traditional approaches to the identification and segmentation of cells are [12]: intensity based, region based, active contours / level sets, probabilistic methods and graphical models.

Methods, analysing the brightness histogram, search for the optimal brightness value, which would limit the pixels within the cell from the background pixels [13].

Methods of region growth can also use the brightness values of pixels, while following the principles of merging the neighbouring regions if they meet pre-determined criteria for a merger [14]

Methods of edge detection splines outline the contour of the nuclei (and other shells) using a gradient image information [15].

Probabilistic models (e.g. Gaussian mixture models [16]) represent cells as a weighted sum of the densities of a normal (Gaussian) distribution, or as a combination of densities of the normal and the other (e.g. gamma) distributions. The parameters of these models are usually estimated on the basis of training data using the methods of estimation of the parameters, such as the EM-algorithm (Expectationmaximization algorithm) [16].

The methods of cutting the graph present the original image as a weighted undirected graph in which each pixel is a node, and the relationship between the nodes form the ribs. These methods divide the graph into disjoint sub-graphs so that the similarity of nodes is high, and it is low in the different subgraphs [17].

\section{It is possible to combine several of the above approaches.}

Otsu method [18] allows to divide the pixels into two classes ("useful" and "background") calculating a threshold where the interclass variance is minimal.

Watershed clustering considers the absolute value of the gradient image as a topographic surface. Pixels, which have the largest absolute value of the brightness gradient, correspond to the lines of the watershed, which represent the boundaries of the regions. The water is placed on any pixel within the general line of the watershed, flows down to a common local minimum brightness. Pixels on which the water drains to a common minimum, columbine form, which is the segment [19]. 
While segmenting the various parts of the cells, it may be effective to combine different algorithms to separate different areas, e.g. K-mean clustering [20] for the segmentation of the nuclei and Fuzzy C-mean clustering [21] for the segmentation of the cytoplasm [22].

It is also possible to represent an image as a probability map, where each pixel stores a probability of belonging to the boundary cells on the basis of a given classifier, followed by the area segmentation using correlation clustering [23, 24].

To perform segmentation of images obtained by the methods of computed tomography, the ITK Insight Toolkit [25] can be applied.

In addition, an ilastic [26] software tool provides possibilities to classify, segment and analyse the objects on the image. This tool has functions for automatic pixel and object classification, automatic and semi-automatic object tracking, semi-automatic segmentation and object counting without its recognition.

\section{MODELling THE XENOPUS LAEVIS EMBRYO}

Images of Xenopus Laevis embryo were obtained at European Synchrotron Research Facility (ESRF) and Advanced Photon Source (APS) using X-Ray phase contrast [2] and were provided by the Institute of Photonic Research and synchrotron radiation (IPS), Technology Institute of Karlsruhe, Karlsruhe, Germany.

Data are presented as a set (stack) of TIFF images wherein each picture is a cut of embryo three-dimensional model with a certain pitch in the vertical axis (Fig. 1).

Fig. 1 shows three types of cells: ectoderm - rounded cells on the surface of the embryo, mesoderm - densely arranged small cells within the embryo, endoderm - large cells within the embryo which are at a big distance from one another.

At the initial stage, segmentation of the original image and selection of cells that belong to different types were performed (Fig. 2).

Generating cellular structures was carried out using autoPACK and Cinema 4D software.

AutoPACK is designed for packaging objects inside the volume and on the surface of the container, using the algorithms that minimize or completely eliminate the overlap.

The basic concepts of this software are a scene, an ingredient, a container and a recipe. The scene includes objects: lights, cameras, geometries, materials and effects. The ingredient is a building block that repeatedly appears in the model. While placing the ingredient, main task is to control the packing density and the elimination of intersections. The ingredients are placed inside- the container or on its surface. Set of packing parameters (including molar mass and concentration, packing algorithms, a description of geometric primitives, constituent ingredients and their location) is called recipe [27, 28].

It is possible to list the five main methods of packing that the software provides: jitter, spring, rigit-body, pandaBullet
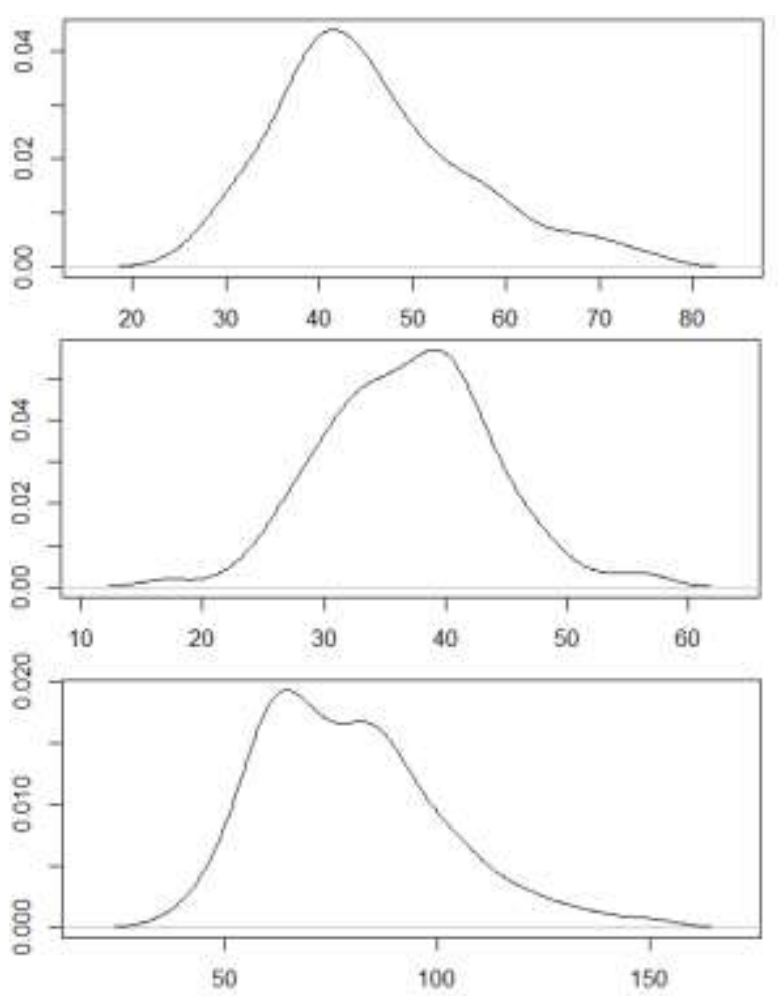

Fig. 2. Density distribution of cell size for ectoderm, mesoderm and endoderm (from top to bottom). The horizontal axis indicates the size of the cells in microns. The vertical axis indicates the density value/

and pandaBulletRelax - where jitter is the least demanding computational algorithm.

Jitter allocates random components on the grid, reducing collisions with other objects, but not eliminating them. The rest of the algorithms collect information about nearby objects and use it to carry out several attempts to place the object, so that is belonged to the other group, excluding the intersection of surfaces. There is integration with the image editors (for example, Panda 3D).

The simulation was performed on a computer having sixcore processor Intel Core i7-3930K, the amount of RAM of 64 GB and the GeForce GTX 680 video card.

Because of the huge number of cells in the complete model ( 109 cells), the generating takes long time intervals, that is the reason to divide the ectoderm and the mesoderm into 24 sections (Fig. 3).

The process of packing one sector with mesoderm or ectoderm cells takes 15-20 minutes. Packing with endoderm cells took up less than 5 minutes for a single pass.

Total assembly time was about 8 hours without rendering.

Next step is rendering of three-dimensional model which was carried out in Cinema 4D (Fig. 4) using Ambient Occlusion, Depth Of Field, Object Glow and other effects.

Rendering a single image takes about 30 minutes.

It should be noted that the reduction of the cell number by 8 times decreases time intervals for packaging approximately 3-4 


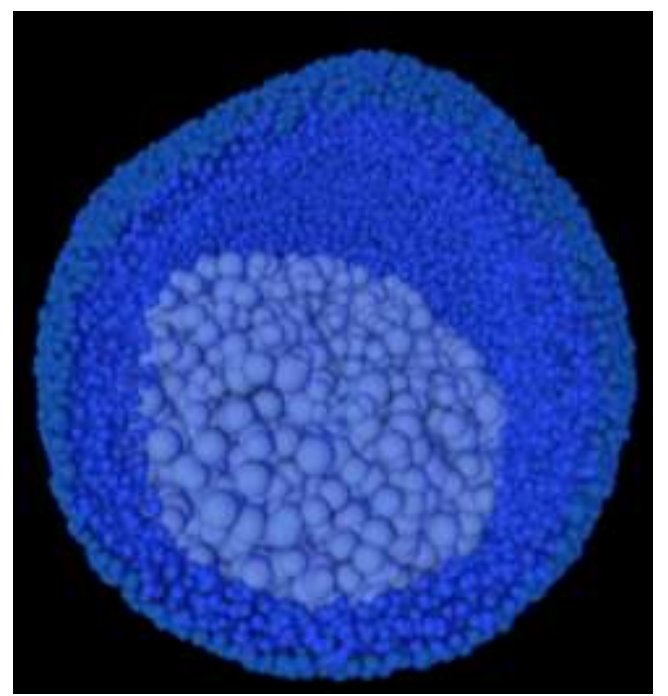

Fig. 3. Three-dimensional model of the embryo, visualized in Cinema 4D (the area of the ectoderm, mesoderm and endoderm are marked with different colours).

times. That makes editing and preview of the reduced model to be carried out much faster. While the embryo is on gastrulation stage, when the process of differentiation of tissues starts, it means, on the subsequent stages the number of its cells will increase, causing the need to attract more computational resources for data processing.

\section{CONCLUSION}

The sphere of programming and algorithmization, considering problems of identification of the mathematical and statistical relationships required for modelling the cellular structures, is currently on the stage of formation [29]. Software products for modelling cellular processes and cell packaging are being developed and improved, but they are not able to provide a solution for all the problems that arise, even though their current versions can build simple cell structures. Their disadvantage is that they are not designed to generate a huge number of cells forming tissues and organs.

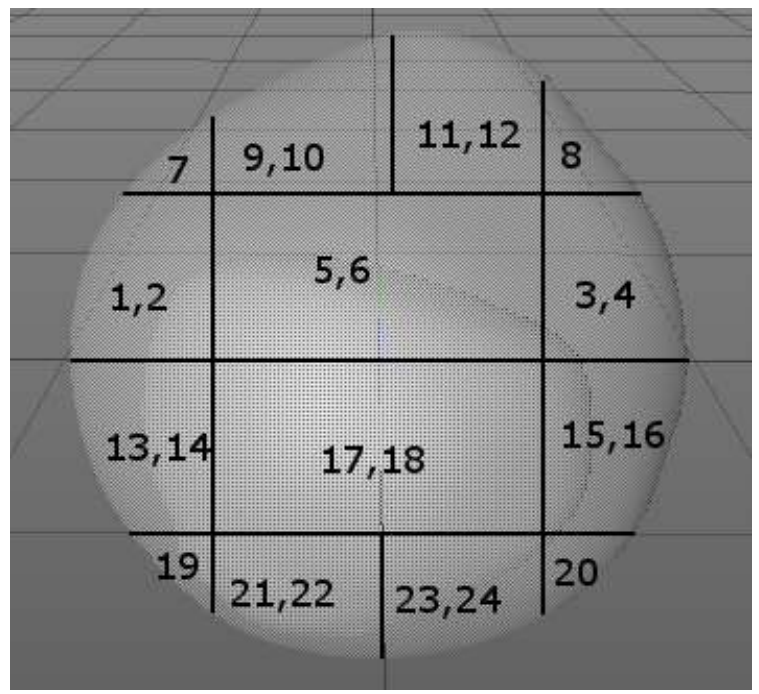

Fig. 4. Embryo model divided into 24 regions.

\section{REFERENCES}

[1] Tinsley, R., Minter, L., Measey, J., Howell, K., Veloso, A., Núñez, H. \& Romano, A. (2009). "Xenopus laevis". IUCN Red List of Threatened Species. Version 2013.2. International Union for Conservation of Nature. Retrieved 4 May 2014.

[2] Moosmann, Julian, Ershov, Alexey, Altapova, Venera, Baumbach, Tilo, Prasad, Maneeshi S., LaBonne, Carole, Xiao, Xianghui, Kashef, Jubin and Hofmann, Ralf. "X-ray phase-contrast in vivo microtomography probes new aspects of Xenopus gastrulation." Nature 497 , no. 7449 (2013): 374--377.

[3] Cyril Petibois. "Imaging methods for elemental, chemical, molecular,and morphological analyses of single cells", Analytical and bioanalytical chemistry, Springer, Berlin, DE, vol. 397, no. 6, 19 March 2010 (2010-03-19), pages 2051-2065, XP019839160, ISSN: 1618-2650

[4] Abramowitz M, Davidson MW (2007). "Introduction to Microscopy". Molecular Expressions.

[5] Introduction to Fluorescence Microscopy, Kenneth R. Spring, Michael W. Davidson, 2000

[6] O1 Optical Microscopy By Katarina Logg. Chalmers Dept. Applied Physics. 2006-01-20.

[7] Weon, B. M.; Je, J. H.; Margaritondo, G. (2006). "Phase contrast X-ray imaging". International Journal of Nanotechnology 3 (2-3): 280-297.

[8] Lang, K.M.; D. A. Hite; R. W. Simmonds; R. McDermott; D. P. Pappas; John M. Martinis (2004). "Conducting atomic force microscopy for nanoscale tunnel barrier characterization". Review of Scientific Instruments 75 (8): 2726-2731. Bibcode:2004RScI...75.2726L. doi:10.1063/1.1777388.

[9] K. Hinrichs and K.-J. Eichhorn (Editors), Ellipsometry of Functional Organic Surfaces and Films, Springer (2014), ISBN 978-3-642-40128-2

[10] Bjorn Kemper; Patrik Langehanenberg; Gert von Bally (2007). "Digital Holographic Microscopy: A New Method for Surface Analysis and Marker?Free Dynamic Life Cell Imaging". Optik \& Photonik (2): 4144.

[11] Gerry McDermott, Mark A. Le Gros, Christian G. Knoechel, Maho Uchida, and Carolyn A. Larabell. "Soft X-ray Tomography and Cryogenic Light Microscopy: The Cool Combination in Cellular Imaging."

[12] Cell words: Modelling the visual appearance of cells in histopathology images. K. Sirinukunwattana, A.M. Khan, and N. M. Rajpoot, Computerized Medical Imaging and Graphics, Elsevier. doi: 10.1016/j.compmedimag. 2014.11.008

[13] Pham, Dzung L.; Xu, Chenyang; Prince, Jerry L. (2000). "Curren Methods in Medical Image Segmentation". Annual Review of Biomedical Engineering 315-337. doi:10.1146/annurev.bioeng.2.1.315. PMID 11701515.

[14] D. Bader, 9. JaJJB, D. Harwood, and L. Davis. Parallel Algorithms for Image Enhancement and Segmentation by Region Growing with an Experimental Study. Technical Report CSTR-3449 and UMIACS-TR95-44, Institute for Advanced Computer Studies (UMIACS), University of Maryland, College Park, MD, May 1995. To be presented at the 10th International Parallel Processing Symposium, Honolulu, HI, April 15 19, 1996.

[15] Lindeberg, Tony (2001), "Edge detection", in Hazewinkel, Michiel, Encyclopedia of Mathematics, Springer, ISBN 978-1-55608-010-4

[16] Dinov, ID. "Expectation Maximization and Mixture Modeling Tutorial". California Digital Library, Statistics Online Computational Resource, Paper EM_MM

[17] Andreev, Konstantin and Räcke, Harald, (2004). "Balanced Graph Partitioning". Proceedings of the sixteenth annual ACM symposium on Parallelism in algorithms and architectures (Barcelona, Spain): 120-124. doi:10.1145/1007912.1007931. ISBN 1-58113-840-7.

[18] M. Sezgin and B. Sankur (2004). "Survey over image thresholding techniques and quantitative performance evaluation". Journal of Electronic Imaging 13 (1): 146-165. doi:10.1117/1.1631315.

[19] Luc Vincent and Pierre Soille. Watersheds in digital spaces: an efficient algorithm based on immersion simulations. In IEEE Transactions on Pattern Analysis and Machine Intelligence, Vol. 13, Num. 6 (1991), pages 583-598. 
[20] Kanungo, T.; Mount, D. M.; Netanyahu, N. S.; Piatko, C. D.; Silverman, R.; Wu, A. Y. (2002). "An efficient k-means clustering algorithm: Analysis and implementation". IEEE Trans. Pattern Analysis and Machine Intelligence 24: 881-892.

[21] Forghani, M.; Forouzanfar, M.; Teshnehlab, M. (2010). "Parameter optimization of improved fuzzy c-means clustering algorithm for brain MR image segmentation". Engineering Applications of Artificial Intelligence 23 (2): 160-168.

[22] Sedat Nazlibilek, Deniz Karacor, Tuncay Ercan, Murat Husnu Sazli, Osman Kalender,Yavuz Ege. "Automatic segmentation, counting, size determination and classification of white blood cells.", Elsevier Press, 2014

[23] Becker, Hila, "A Survey of Correlation Clustering", 5 May 2005.

[24] Chong Zhang, Julian Yarkony, and Fred A. Hamprecht. "Cell Detection and Segmentation using Correlation Clustering". Medical Image Computing and Computer-Assisted Intervention - MICCAI 2014. Volume 8673 of the series Lecture Notes in Computer Science pp 9-16

[25] Yoo, TS; Ackerman, MJ; Lorensen, WE; et al. (2002). "Engineering and algorithm design for an image processing Api: a technical report on ITK - the Insight Toolkit". Stud Health Technol Inform 85: 586-92. PMID 15458157

[26] Sommer, C; Straehle C; Koethe U; Hamprecht FA (2011). "Ilastik: Interactive Learning and Segmentation Toolkit". IEEE International Symposium on Biomedical Imaging: 230-33. doi:10.1109/ISBI.2011.5872394

[27] Johnson G, Autin L, Al-Alusi M, Goodsell D, Sanner M, Olson A. autoPACK. LGPL open-source, autoPACK.org, UCSF and The Scripps Research Institute, 2006-2013.

[28] Tsapko I. V., Vlasov A. V. Registration Process of Geometric Scan Data of an Object [Electronic resources] // Mechanical Engineering, Automation and Control Systems (MEACS) : proceedings of the International Conference, Tomsk, 1-4 December, 2015 / National Research Tomsk Polytechnic University (TPU) ; Institute of Electrical and Electronics Engineers (IEEE). - [S. 1.]: IEEE, 2015. - [4 p.].

[29] GPU-optimized Direct Fourier Method for On-line Tomography [Electronic resource] / Roman Shkarin, Evelina Ametova, Suren Chilingaryan, Timo Dritschler, Andreas Kopmann, Alessandro Mirone, Andrei Shkarin, Matthias Vogelgesang, Sergey Tsapko // Fundamenta Informaticae. — 2015. — Vol. 141, iss. 2-3. - [P. 245-258]. 
